\title{
Lignes directrices de la SSR pour l'expertise médicale des maladies rhumatismales et des séquelles rhumatismales d'accidents
}

Société suisse de rhumatologie, groupe de travail «médecine d'assurance», J. Jeger
Correspondance:

Dr Jörg Jeger

Spécialiste en rhumatologie FMH,

EMBA

Médecin-chef MEDAS Zentralschweiz

Zürichstrasse 14

CH-6004 Lucerne

joerg.jeger@medaslu.ch
Les lignes directrices sont des recommandations destinées à guider une action. Elles servent à harmoniser la prestation d'un service, et donc à en garantir la qualité. Dans le système de santé, l'usage des lignes directrices s'est répandu dans les domaines les plus variés sous l'influence de la médecine fondée sur les preuves. Ce phénomène peut s'expliquer par de nombreuses raisons, notamment par les exigences croissantes en matière de sécurité des patients et la diminution des ressources dont dispose la société.

Entre les années 1990 et 2002, on a assisté à une augmentation dramatique des demandes de prestations d'assurance, notamment des demandes de rentes formulées auprès de l'assuranceinvalidité. Depuis 2003, les chiffres affichent de nouveau une régression. Les troubles psychiques et les maladies de l'appareil locomoteur représentant depuis longtemps les causes principales. Tandis que chez les patients les plus jeunes, les troubles psychiques prédominent, chez les patients âgés de plus de 55 ans, ce sont les maladies de l'appareil locomoteur qui sont la cause la plus fréquente d'une rente anticipée [1]. Les atteintes de l'appareil locomoteur sont généralement évaluées par des rhumatologues, qui sont responsables de leur expertise. Force est de constater qu'en Suisse, les spécialistes bien formés capables de réaliser ces expertises sont en nombre insuffisant.

En 2004, la Société suisse de psychiatrie d'assurance a publié des lignes directrices pour l'expertise médicale des troubles psychiques [2] qui ont eu une grande résonance, et auxquelles le Tribunal fédéral des assurances se réfère désormais parfois dans ses arrêts [3]. A son tour, le groupe de travail «médecine d'assurance» de la Société suisse de rhumatologie publie des lignes directrices pour l'expertise médicale des maladies rhumatismales et des séquelles rhumatismales d'accidents, que nous présentons ici. Ce groupe de travail se compose des membres suivants:

- Pr Dr Peter Villiger, directeur de la clinique de rhumatologie à l'Hôpital de l'Ile de Berne, président de la Société suisse de rhumatologie;
- Dr Pius Brühlmann, médecin dirigeant, clinique de rhumatologie de l'Hôpital universitaire de Zurich;

- Dr Dieter Frey, rhumatologue, Bâle;

- Dr Thomas Meyer, médecin dirigeant rhumatologie, Hôpital cantonal de Baden;

- Dr Hans Schwarz, médecin-chef rhumatologie, Hôpital Bethesda de Bâle;

- Dr Jörg Jeger, médecin-chef MEDAS Zentralschweiz (directeur du groupe de travail).

Ces lignes directrices ont été approuvées par le comité de la Société suisse de rhumatologie lors de la séance du 27 septembre 2006. Elles sont destinées à aider tous les rhumatologues ayant une activité d'expertise, mais aussi les médecins qui suivent une formation pour devenir rhumatologue.

L'activité d'expertise, à mi-chemin entre la médecine et le droit appliqué, requiert à bien des égards un vocabulaire spécifique souvent difficile à comprendre pour le néophyte, et qui peut occasionner des malentendus. Pour cette raison, nous avons intégré un glossaire complet expliquant les concepts les plus courants qui constituent le bagage dont l'expert a besoin dans le cadre de son activité. Juristes et médecins doivent pouvoir se comprendre en dépit des manières différentes - déductive dans un cas, inductive dans l'autre - dont ils envisagent et apprécient une situation. Nous tenons à remercier cordialement M. Pr Dr iur. U. Meyer, juge fédéral au Tribunal fédéral des assurances de Lucerne, qui a bien voulu relire en juriste les présentes lignes directrices et leur apporter des corrections.

\section{Références}

1 Office fédéral des assurances sociales. Statistique de l'AI 2006. www.bsv.admin.ch.

2 Lignes directrices de la Société suisse de psychiatrie d'assurance pour l'expertise médicale des troubles psychiques. Bull Méd Suisses. 2004;85(36):1905-9.

3 Arrêt du Tribunal fédéral des assurances U 391/06 du 9.8.2006. www.bger.ch. 